\title{
Bio-Intensive Management of Thrips Transmitted Bud Necrosis Disease in Tomato
}

\author{
H.D. Vinaykumar ${ }^{*}$, M.R. Govindappa ${ }^{2}$ and Y.B. Naveesh ${ }^{3}$ \\ ${ }^{1}$ Department of Plant Pathology, UAS Raichur, Karnataka 584104, India \\ ${ }^{2}$ Agriculture extension and education centre, Koppal, Karnataka, India \\ ${ }^{3}$ Department of Plant Pathology, UAS, Bengaluru, Karnataka 560065, India \\ *Corresponding author
}

\section{A B S T R A C T}

\section{Keywords \\ Peanut Bud Necrosis Disease (PBND), Peanut Bud Necrosis Virus (PBNV) and Thiamethoxam \\ Article Info \\ Accepted: 10 March 2018 Available Online: 10 April 2018}

In order to evaluate the efficacy of new chemical molecules, bio agent and botanicals on management of Peanut bud necrosis disease (PBND) in tomato, field experiment was conducted with different management practices during Kharif 2014 at MARS. Among the different chemical schedule the best schedule was $\mathrm{T}_{5}$ (seedling dip with imidachloprid 17.8 SL at.03ml/1 - P. fluorescens @ 5g/l - Thiamethoxam $0.2 \mathrm{~g} / \mathrm{l}$ - Neemoil (1500 ppm)@ $5 \mathrm{ml} / \mathrm{l}$ at 15 days interval from planting) with the disease reduction of 50.00 per cent and with a yield of $29.12 \mathrm{t} / \mathrm{ha}$.

\section{Introduction}

Tomato [Solanum lycopersicum (Mill.) Wettsd] is the second most important vegetable crop after potato and belongs to the family Solanaceae. In India it is grown on 905.5 ('000 ha) with the production of 19103.99 ('000 MT) with an average yield of 20.8 (MT/ha) (Anon., 2014).

Though, the area under tomato cultivation is high, the productivity (20 tones/ha) is low, due to biotic factors like insect pests and diseases. Among the diseases caused by fungi and bacteria it is also affected by large number of viral diseases (Anon., 1983). Tomato is reported to be susceptible to over 40 viruses belonging to Alfamo, Luteo, Carla, Cucumo, Gemini, Poty, Illar, Nepo, Tombus, Tobamo and Tospovirus groups (Allen and Gibbs, 1990). Among several viral diseases of tomato bud necrosis disease of tomato is predominant ones. This disease is caused by Peanut bud necrosis virus (PBNV) belonging to genus Tospovirus the only plant infecting genus in the family Bunyaviridae and transmitted by thrips (Thrips plami Karmy). As the disease is transmitted by thrips reducing the thrips populations using appropriate insecticides can help to reduce the virus spread. However, 
insecticides alone are of limited value in insect transmitted viral disease management, as virus spread from non-crop areas is an important source of infection and thrips require only limited feeding times for virus transmission. Frequent use of insecticides may also lead to development of insecticide resistance in thrips populations. Hence, strategic planning in the management of by using the botanicals like neemoil and biocontrol agents like Pseudomonas fluorescent would offer great scope in the management of virus diseases by inducing resistance in plants. With this aim, the present study was conducted to manage the bud necrosis disease in tomato by using botanicals, biocontrol agent and insecticides.

\section{Materials and Methods}

A field experiment with tomato variety Arka vikas was laid out in Main agriculture research station (MARS), University of Agriculture Sciences, Raichur during Kharif 2014 to evaluate the efficacy of different treatment combination in reducing the incidence of bud necrosis disease of tomato. Eight treatments (Table 3) were replicated thrice in Randomized block design. Till the time of transplanting the seedling were protected under nylon net (50 mesh).The per cent disease incidence was calculated by using the below mentioned formula. The observation on per cent disease incidence was recorded at before $1^{\text {st }}, 2^{\text {nd }}$, and $3^{\text {rd }}$ spray and 15 days after $3^{\text {rd }}$ spray and the data were analysed statistically. The per cent disease reduction over control was calculated by using the formula given by Vincent (1927).

$$
\text { PDI }(\%)=\frac{\text { Number of diseased plants }}{\text { Total number of plants }}
$$

Per cent disease reduction $(\%)=\frac{(\mathrm{C}-\mathrm{T})}{\mathrm{C}}$

\section{Results and Discussion}

In the current study efficacy of new chemicals, bio agent ( $P$. fluorescens) and botanicals (neem oil) on the incidence of PBNV disease on tomato revealed that during the time of planting, none of the seedlings showed PBND incidence as they were protected under nylon net $(50 \mathrm{mesh})$. In parallel to this, delay in spread of vector mediated leaf curl virus disease in tomato upto five weeks when 25 days old seedlings protected under nylon mesh (40-50 mesh) transplanted in the field (Muniyappa and Saikia, 1983; Ganesh Naik and Muniyappa, 2004). Further in the present study, seedling dip with imidacloprid (17.8 SL @ $0.3 \mathrm{ml} / \mathrm{l}$ ) showed negligible disease incidence of PBNV, similar opinion was also expressed by Krishnakumar et al., (2006) who stated that seed treatment using imidacloprid (Gaucho $75 \mathrm{WS} \mathrm{3g/} \mathrm{kg} \mathrm{of} \mathrm{seed)} \mathrm{significantly}$ reduced thrips infestation but not WBNV incidence. The results concluded that management of thrips does not effectively translate to WBNV reduction on watermelon. After first spray with Pseudomonas fluorescens, least incidence was recorded in $\mathrm{T}_{2}$ $(25.00 \%)$ and this was on par with other treatment viz., $\mathrm{T}_{5}, \mathrm{~T}_{4}, \mathrm{~T}_{3}$, and $\mathrm{T}_{6}$ with the incidence of $26.39,27.78,27.78$, and 29.17 per cent respectively and highest disease incidence was recoded in $\mathrm{T}_{8}$ (untreated check) 36.11 per cent (Table 1). Among treatments, no significant difference was observed, except untreated check which differed significantly. The slight reduction of disease incidence in treatments over the control may be due to activation of defence related enzymes, this similar opinion was also expressed by Sanjay and Sivasubramanian (2012) who reported that the activity of defence related enzymes viz., Peroxidase (POD), Polyphenoloxidase (PPO) and Phenylalanine Ammonia Lyase (PAL) was enhanced in onion plants treated with foliar application of Pseudomonas fluorescens. 
Table.1 Effect of different management practices on the incidence of bud necrosis disease and yield of tomato under field condition during Kharif 2014

\begin{tabular}{|c|c|c|c|c|c|c|c|}
\hline \multirow{2}{*}{$\begin{array}{l}\text { Tr. } \\
\text { No. }\end{array}$} & \multirow[t]{2}{*}{ Treatments details } & \multicolumn{4}{|c|}{ Per cent disease incidence at } & \multirow{2}{*}{$\begin{array}{l}\text { Yield } \\
(\mathrm{t} / \mathrm{ha})\end{array}$} & \multirow{2}{*}{$\begin{array}{l}\text { Per cent disease } \\
\text { reduction over } \\
\text { control }\end{array}$} \\
\hline & & $\begin{array}{c}15 \\
\text { DAT }\end{array}$ & $\begin{array}{c}30 \\
\text { DAT }\end{array}$ & $\begin{array}{l}45 \\
\text { DAT }\end{array}$ & 60 DAT & & \\
\hline $\mathbf{T}_{1}$ & Seedling dip with Imidacloprid $17.8 \mathrm{SL}$ at $0.3 \mathrm{ml} / 1$ treatment & $\begin{array}{c}4.17 \\
(11.77)\end{array}$ & $\begin{array}{c}31.94 \\
(34.37)\end{array}$ & $\begin{array}{c}55.56 \\
(48.17)\end{array}$ & $\begin{array}{c}61.11 \\
(51.43)\end{array}$ & 17.18 & 11.99 \\
\hline $\mathbf{T}_{2}$ & $\begin{array}{l}\mathrm{T} 1-P . \text { fluorescens at } 5 \mathrm{~g} / \mathrm{l}-\text { Imidacloprid } 17.8 \mathrm{SL} \text { at } 0.3 \mathrm{ml} / \mathrm{l}- \\
\text { Neemoil }(1500 \mathrm{ppm}) \text { at } 5 \mathrm{ml} / \mathrm{l} \text { at } 15 \text { days interval from planting }\end{array}$ & $\begin{array}{c}4.17 \\
(11.77)\end{array}$ & $\begin{array}{c}25.00 \\
(29.77)\end{array}$ & $\begin{array}{c}48.61 \\
(44.19)\end{array}$ & $\begin{array}{c}52.78 \\
(46.60)\end{array}$ & 21.35 & 23.99 \\
\hline $\mathbf{T}_{3}$ & $\begin{array}{l}\text { T1- P. fluorescens at } 5 \mathrm{~g} / \mathrm{l}-\text { Diafenthurion } 50 \mathrm{WP} \text { at } 1 \mathrm{~g} / \mathrm{l}- \\
\text { Neemoil }(1500 \mathrm{ppm}) \text { at } 5 \mathrm{ml} / \mathrm{l} \text { at } 15 \text { days interval from planting }\end{array}$ & $\begin{array}{c}5.56 \\
(13.44)\end{array}$ & $\begin{array}{c}27.78 \\
(31.74)\end{array}$ & $\begin{array}{c}45.83 \\
(42.57)\end{array}$ & $\begin{array}{c}50.00 \\
(44.99)\end{array}$ & 23.10 & 27.99 \\
\hline $\mathbf{T}_{4}$ & $\begin{array}{l}\mathrm{T} 1-\text { P. fluorescens } 5 \mathrm{~g} / \mathrm{l} \text { - Fipronil } 5 \% \mathrm{SC} \text { at } 1 \mathrm{ml} / \mathrm{l}-\text { Neemoil } \\
(1500 \mathrm{ppm}) 5 \mathrm{ml} / \mathrm{l} \text { at } 15 \text { days interval from planting }\end{array}$ & $\begin{array}{c}4.17 \\
(11.77)\end{array}$ & $\begin{array}{c}27.78 \\
(31.50)\end{array}$ & $\begin{array}{c}43.06 \\
(40.94)\end{array}$ & $\begin{array}{c}47.22 \\
(43.37)\end{array}$ & 24.07 & 31.99 \\
\hline $\mathbf{T}_{5}$ & $\begin{array}{l}\mathrm{T} 1-P . \text { fluorescens at } 5 \mathrm{~g} / \mathrm{l} \text { - Thiamethoxam } 0.2 \mathrm{~g} / \mathrm{l} \text { - Neemoil } \\
(1500 \mathrm{ppm}) \text { at } 5 \mathrm{ml} / \mathrm{l} \text { at } 15 \text { days interval from planting }\end{array}$ & $\begin{array}{c}4.17 \\
(11.77)\end{array}$ & $\begin{array}{c}26.39 \\
(30.88)\end{array}$ & $\begin{array}{c}30.56 \\
(33.50)\end{array}$ & $\begin{array}{c}34.72 \\
(36.06)\end{array}$ & 29.12 & 50.00 \\
\hline $\mathbf{T}_{6}$ & $\begin{array}{l}\text { T1- P. fluorescens at } 5 \mathrm{~g} / \mathrm{l}-\mathrm{Dinotefuron} 20 \mathrm{SG} \text { at } 0.2 \mathrm{~g} / 1- \\
\text { Neemoil }(1500 \mathrm{ppm}) \text { at } 5 \mathrm{ml} / \mathrm{l} \text { at } 15 \text { days interval from planting }\end{array}$ & $\begin{array}{c}4.17 \\
(11.77)\end{array}$ & $\begin{array}{c}29.17 \\
(32.44)\end{array}$ & $\begin{array}{c}33.33 \\
(35.22)\end{array}$ & $\begin{array}{c}37.50 \\
(37.67)\end{array}$ & 26.03 & 45.99 \\
\hline $\mathbf{T}_{7}$ & $\begin{array}{l}\text { P. fluorescens at } 5 \mathrm{~g} / \mathrm{l} \text { - Imidacloprid } 17.8 \mathrm{SL} \text { at } 0.3 \mathrm{ml} / 1 \\
\text { Neemoil }(1500 \mathrm{ppm}) \text { at } 5 \mathrm{ml} / \mathrm{l} \text { - at } 15 \text { days interval from planting }\end{array}$ & $\begin{array}{c}6.94 \\
(15.11)\end{array}$ & $\begin{array}{c}33.33 \\
(35.22)\end{array}$ & $\begin{array}{c}54.17 \\
(47.39)\end{array}$ & $\begin{array}{c}58.33 \\
(49.79)\end{array}$ & 18.98 & 15.99 \\
\hline $\mathbf{T}_{8}$ & Untreated control & $\begin{array}{c}8.33 \\
(16.41)\end{array}$ & $\begin{array}{c}36.11 \\
(36.87)\end{array}$ & $\begin{array}{c}65.28 \\
(53.99)\end{array}$ & $\begin{array}{c}69.44 \\
(56.58)\end{array}$ & 12.29 & \\
\hline & S.Em \pm & 1.32 & 2.82 & 2.61 & 2.68 & 1.34 & \\
\hline & C.D. at $5 \%$ & 4.01 & 8.56 & 7.91 & 8.14 & 4.05 & \\
\hline
\end{tabular}


Table. 2 Cost economics of bio-intensive management of thrips transmitted bud necrosis disease in tomato

\begin{tabular}{|c|c|c|c|c|c|c|c|}
\hline Treatments & $\begin{array}{l}\text { Cost of } \\
\text { cultivation(Rs./ha) } \\
\text { A }\end{array}$ & $\begin{array}{c}\text { Cost of plant } \\
\text { protection(Rs./ha) } \\
\text { B }\end{array}$ & $\begin{array}{l}\text { Total cost of } \\
\text { production(Rs./ha) } \\
(\mathrm{A}+\mathrm{B})\end{array}$ & Yield(kg/ha) & $\begin{array}{l}\text { Gross return } \\
\text { (Rs./ha) }\end{array}$ & $\begin{array}{l}\text { Net } \\
\text { returns(Rs./ha) }\end{array}$ & $\mathrm{B}: \mathrm{C}$ \\
\hline $\begin{array}{l}\mathrm{T}_{1=} \text { Seedling dip with } \\
\text { Imidacloprid } 17.8 \mathrm{SL} \text { at } 0.3 \mathrm{ml} / \mathrm{l}\end{array}$ & $1,20,000$ & 3017 & 123017 & 17180 & 343600 & 220583 & 2.79 \\
\hline $\begin{array}{l}\mathrm{T}_{2=} \mathrm{T}_{1}-P \text {. fluorescens at } 5 \mathrm{~g} / \mathrm{l}- \\
\text { Imidacloprid } 17.8 \mathrm{SL} \text { at } 0.3 \mathrm{ml} / \mathrm{l} \\
- \text { Neemoil }(1500 \mathrm{ppm}) \text { at } 5 \mathrm{ml} / \mathrm{l} \\
\text { at } 15 \text { days interval from } \\
\text { transplanting }\end{array}$ & $1,20,000$ & 10596.5 & 130596.5 & 21350 & 427000 & 296403.5 & 3.26 \\
\hline $\begin{array}{l}\mathrm{T}_{3}=\mathrm{T}_{1}-P \text {. fluorescens at } 5 \mathrm{~g} / \mathrm{l}- \\
\text { Diafenthurion } 50 \mathrm{WP} \text { at } 1 \mathrm{~g} / \mathrm{l} \text { - } \\
\text { Neemoil }(1500 \mathrm{ppm}) \text { at } 5 \mathrm{ml} / \mathrm{l} \text { at } \\
15 \text { days interval from } \\
\text { transplanting }\end{array}$ & $1,20,000$ & 9879.5 & 129879.5 & 23100 & 462000 & 332120.5 & 3.55 \\
\hline $\begin{array}{l}\mathrm{T}_{4}=\mathrm{T}_{1}-P . \text { fluorescens at } 5 \mathrm{~g} / \mathrm{l}- \\
\text { Fipronil } 5 \% \mathrm{SC} \text { at } 1 \mathrm{ml} / \mathrm{l}- \\
\text { Neemoil }(1500 \mathrm{ppm}) 5 \mathrm{ml} / \mathrm{l} \text { at } 15 \\
\text { days interval from } \\
\text { transplanting }\end{array}$ & $1,20,000$ & 9579.5 & 129579.5 & 24070 & 481400 & 351820.05 & 3.71 \\
\hline $\begin{array}{l}\mathrm{T}_{5}=\mathrm{T}_{1}-P . \text { fluorescens at } 5 \mathrm{~g} / \mathrm{l} \text { - } \\
\text { Thiamethoxam } 0.2 \mathrm{~g} / \mathrm{l} \text { - Neemoil } \\
(1500 \mathrm{ppm}) 5 \mathrm{ml} / \mathrm{l} \text { at } 15 \text { days } \\
\text { interval from planting }\end{array}$ & $1,20,000$ & 8679.5 & 128679.5 & 29120 & 582400 & 453720.5 & 4.52 \\
\hline $\begin{array}{l}\mathrm{T}_{6}=\mathrm{T}_{1}-P . \text { fluorescens at } 5 \mathrm{~g} / \mathrm{l}- \\
\text { Dinotefuron } 20 \mathrm{SG} \text { at } 0.2 \mathrm{~g} / \mathrm{l}- \\
\text { Neemoil }(1500 \mathrm{ppm}) \text { at } 5 \mathrm{ml} / \mathrm{l} \text { at } \\
15 \text { days interval from } \\
\text { transplanting }\end{array}$ & $1,20,000$ & 9679.5 & 129679.5 & 26030 & 520600 & 390920.5 & 4.01 \\
\hline $\begin{array}{l}\mathrm{T}_{7}=P \text {. fluorescens at } 5 \mathrm{~g} / \mathrm{l}- \\
\text { Imidacloprid } 17.8 \mathrm{SL} \text { at } 0.3 \mathrm{ml} / \mathrm{l} \\
\text { Neemoil }(1500 \mathrm{ppm}) \text { at } 5 \mathrm{ml} / \mathrm{l}-\mathrm{at} \\
15 \text { days interval from } \\
\text { transplanting }\end{array}$ & $1,20,000$ & 13096.5 & 133096.5 & 18980 & 379600 & 246503.5 & 2.82 \\
\hline $\mathrm{T}_{8}=$ Untreated check & $1,20,000$ & 0 & 120000 & 12290 & 245800 & 125800 & 2.04 \\
\hline
\end{tabular}

Market price of tomato fruit at harvest period (2014): Rs 20. $/ \mathrm{kg}$, P. fluorescens= Rs 150/kg, Neem oil= Rs 250/100ml, Imidacloprid= Rs 530/100ml,

Diafenthurion= Rs1600/kg, Fipronil= Rs650/500ml, Thiamethoxam= Rs 650/kg, Dinotefuron= Rs 800/kg 
Table.3 Details of treatments of the experiment

\begin{tabular}{|c|c|c|}
\hline Sl. No. & Treatments & Description \\
\hline 1 & $\mathrm{~T}_{1}$ & Seedling dip with Imidacloprid $17.8 \mathrm{SL}$ at $0.3 \mathrm{ml} / \mathrm{l}$ \\
\hline 2 & $\mathrm{~T}_{2}$ & $\begin{array}{l}\mathrm{T} 1-P . \text { fluorescens at } 5 \mathrm{~g} / \mathrm{l}-\text { Imidacloprid } 17.8 \mathrm{SL} \text { at } 0.3 \mathrm{ml} / \mathrm{l}-\text { Neemoil } \\
(1500 \mathrm{ppm}) \text { at } 5 \mathrm{ml} / \mathrm{l} \text { at } 15 \text { days interval from transplanting }\end{array}$ \\
\hline 3 & $\mathrm{~T}_{3}$ & $\begin{array}{l}\mathrm{T} 1-P \text {. fluorescens at } 5 \mathrm{~g} / \mathrm{l} \text {-Diafenthurion } 50 \mathrm{WP} \text { at } 1 \mathrm{~g} / \mathrm{l} \text {-Neemoil }(1500 \\
\mathrm{ppm}) \text { at } 5 \mathrm{ml} / \mathrm{l} \text { at } 15 \text { days interval from transplanting }\end{array}$ \\
\hline 4 & $\mathrm{~T}_{4}$ & $\begin{array}{l}\mathrm{T} 1-P \text {. fluorescens at } 5 \mathrm{~g} / \mathrm{l} \text {-Fipronil } 5 \% \mathrm{SC} \text { at } 1 \mathrm{ml} / \mathrm{l} \text {-Neemoil }(1500 \mathrm{ppm}) 5 \\
\mathrm{ml} / \mathrm{l} \text { at } 15 \text { days interval from transplanting }\end{array}$ \\
\hline 5 & $\mathrm{~T}_{5}$ & $\begin{array}{l}\mathrm{T} 1-P . \text { fluorescens at } 5 \mathrm{~g} / \mathrm{l} \text { - Thiamethoxam } 0.2 \mathrm{~g} / \mathrm{l} \text { - Neemoil }(1500 \mathrm{ppm}) 5 \\
\mathrm{ml} / \mathrm{l} \text { at } 15 \text { days interval from planting }\end{array}$ \\
\hline 6 & $\mathrm{~T}_{6}$ & $\begin{array}{l}\mathrm{T} 1-P \text {. fluorescens at } 5 \mathrm{~g} / \mathrm{l} \text {-Dinotefuron } 20 \mathrm{SG} \text { at } 0.2 \mathrm{~g} / \mathrm{l} \text { - Neemoil(1500 } \\
\mathrm{ppm}) \text { at } 5 \mathrm{ml} / \mathrm{l} \text { at } 15 \text { days interval from transplanting }\end{array}$ \\
\hline 7 & $\mathrm{~T}_{7}$ & $\begin{array}{l}P \text {. fluorescens at } 5 \mathrm{~g} / \mathrm{l} \text {-Imidacloprid } 17.8 \mathrm{SL} \text { at } 0.3 \mathrm{ml} / \mathrm{l} \mathrm{Neemoil}(1500 \mathrm{ppm}) \\
\text { at } 5 \mathrm{ml} / \mathrm{l} \text {-at } 15 \text { days interval from transplanting }\end{array}$ \\
\hline 8 & $\mathrm{~T}_{8}$ & Untreated control \\
\hline
\end{tabular}

The activity of these enzymes was negatively correlated with thrips population.

After the application of new insecticides as second spray, higher disease incidence was observed in $\mathrm{T}_{8}(65.28 \%)$ and least disease incidence was observed in $\mathrm{T}_{5}(30.56 \%)$ followed by $\mathrm{T}_{6}(33.33 \%)$ at 45 DAT (Table 1$)$.

Similarly, 15 days after the implication of $3^{\text {rd }}$ spray, higher disease incidence was observed in $\mathrm{T}_{8}(69.44 \%)$ and least disease incidence was observed in $\mathrm{T}_{5}(34.72 \%)$ followed by $\mathrm{T}_{6}(37.50$ $\%)$ at 60 DAT (Table 1).

The present study revealed that, among the different chemical schedule, the best schedule was $\mathrm{T}_{5}$ (seedling dip with imidachloprid $17.8 \mathrm{SL}$ at.03ml/1 - P. fluorescens @ 5g/1 Thiamethoxam 0.2 g/l - Neemoil (1500 ppm) @ $5 \mathrm{ml} / \mathrm{l}$ at 15 days interval from planting) with the disease reduction of 50.00 per cent and with a yield of $29.12 \mathrm{t} /$ ha with highest cost benefit ratio of 4.52 (Table 1 and 2).

\section{References}

\section{How to cite this article:}

Vinaykumar, H.D., M.R. Govindappa and Naveesh, Y.B. 2018. Bio-Intensive Management of Thrips Transmitted Bud Necrosis Disease in Tomato. Int.J.Curr.Microbiol.App.Sci. 7(04): 1311-1315. doi: https://doi.org/10.20546/ijcmas.2018.704.146
Allen, B. and Gibbs, K. C., 1990. Viruses of tropical plants. CAB International, Oxon, UK. pp. 707.

Anonymous, 1983. Pest control in tropical tomatoes, Centre for overseas pest research, London, p 130.

Anonymous, 2014. http://agricoop.nic.in/ agristatistics.htm.

Ganesh Naik, R. and Muniyappa, V., 2004. Integrated management of tomato leaf curl gemenivirus disease. Mysore J. Aric. Sci., 38(2): 213-220.

Krishnakumar, N. K., Venkatesh, N., Kalleshwaraswamy, C. M. and Ranganath, H. R., 2006. Seasonal incidence of thrips and bud necrosis virus on water melon. Pest Management in Horticultural Eco-systems., 12: 85-92.

Muniyappa, V. and Saikia, A. K., 1983. Prevention of the spread of tomato leaf curl disease. Indian Phytopath., 36: 183186.

Sanjay Bandi and P. Sivasubramanian, 2012. Management of Thrips tabaci Lindeman in onion using Pseudomonas fluorescens Migula through induced resistance. Journal of Biopesticides. 5: 1-3. 